\title{
TUMOR CARCINOIDE ATÍPICO NASAL. DESCRIPCIÓN DE UN CASO
}

\author{
Nasal atypical carcinoid tumor. A case report \\ Gisela RISSON-PINO; Raquel TUR-GONZÁLEZ; Pablo SANTOS-GORJÓN; Gonzalo MARTIN- \\ HERNÁNDEZ; Elena SÁNCHEZ-TERRADILLOS; Eva MINGO-SÁNCHEZ
}

SACYL. Hospital Nuestra Señora de Sonsoles. Servicio de Otorrinolaringología. Ávila. España

Correspondencia: giselarisson@hotmail.com

Fecha de recepción: 27 de febrero de 2016

Fecha de aceptación: 1 de abril de 2016

Fecha de Publicación: 15 de abril de 2016

Conflicto de intereses: Los autores declaran no tener conflictos de intereses

Imágenes: Los autores declaran haber obtenido las imágenes con el permiso de los pacientes Política de derechos y autoarchivo: se permite el autoarchivo de la versión post-print (SHERPA/RoMEO) Licencia CC BY-NC-ND. Licencia Creative Commons Atribución-NoComercial-SinDerivar 4.0 Internacional (c) Universidad de Salamanca. Su comercialización está sujeta al permiso del editor

RESUMEN Introducción: Los tumores carcinoides son tumores neuroendocrinos que en su mayoría asientan sobre el sistema gastrointestinal, siendo su localización en el área nasosinusal muy infrecuente. Descripción: Se presenta el caso de una mujer de 55 años de edad con una lesión nasal unilateral. El análisis anatomopatológico evidenció características histológicas e inmunohistoquímicas compatibles con tumor carcinoide atípico. Discusión: Los tumores carcinoides pueden ser típicos o atípicos según su grado de diferenciación. Aunque infrecuentes, deben considerarse en el diagnóstico diferencial en poliposis unilaterales. Su tratamiento es esencialmente quirúrgico. Conclusiones: Este caso clínico destaca la importancia del análisis anatomopatológico de todas las lesiones nasales unilaterales.

PALABRAS CLAVE tumor carcinoide; tumor neuroendocrino; senos paranasales

SUMMARY

Introduction: Carcinoid tumors are neuroendocrine tumors that are usually located on the gastrointestinal system, but infrequent in the sinus area. Description: The case of a 55-year-old woman with a unilateral nasal mass is described. The pathological analysis shows histological and immunohistochemical characteristics compatible with atypical carcinoid tumor. Discussion: Carcinoid tumors may be typical or atypical according to their degree of differentiation. Although rare, they should be considered in differential diagnosis of unilateral polyposis. Treatment is essentially surgical. Conclusions: This case highlights the importance of histopathological analysis of all unilateral nasal lesions.

KEYWORDS

carcinoid tumors; neuroendocrine tumors; paranasal sinuses 


\section{INTRODUCCIÓN}

El tumor carcinoide pertenece a una estirpe de tumores de agresividad media o baja. En la mayoría de los casos afectan al sistema gastrointestinal. Otros órganos que pueden verse afectados son pulmones, bronquios, laringe, hígado, páncreas, riñones, próstata y timo [1]. El asiento en el área nasosinusal es infrecuente. Son tumores neuroendocrinos derivados de células madre que pueden secretar hormonas y sustancias vasoactivas provocando efectos sistémicos como el síndrome carcinoide (rubor facial, taquicardia, diarrea, signos respiratorios - sibilancias y broncoespasmos - y alteración del estado de la consciencia con compromiso de la vida del paciente en los casos graves).

A pesar de la baja incidencia de estos tumores en la cavidad nasal deben tenerse en cuenta en el diagnóstico diferencial de las masas nasales unilaterales.

\section{DESCRIPCIÓN}

Mujer de 55 años sin antecedentes patológicos, valorada en consulta de Otorrinolaringología por insuficiencia respiratoria nasal de varios meses de evolución de predominio derecho sin otra sintomatología asociada. La endoscopia nasal evidenció poliposis nasal derecha. En la tomografía axial computarizada de senos paranasales (TAC) se confirmó la tumoración polipoide sin signos radiológicos de agresividad, desmineralización del cornete medio derecho y ocupación pansinusal derecha por obstrucción del drenaje (Figura 1).

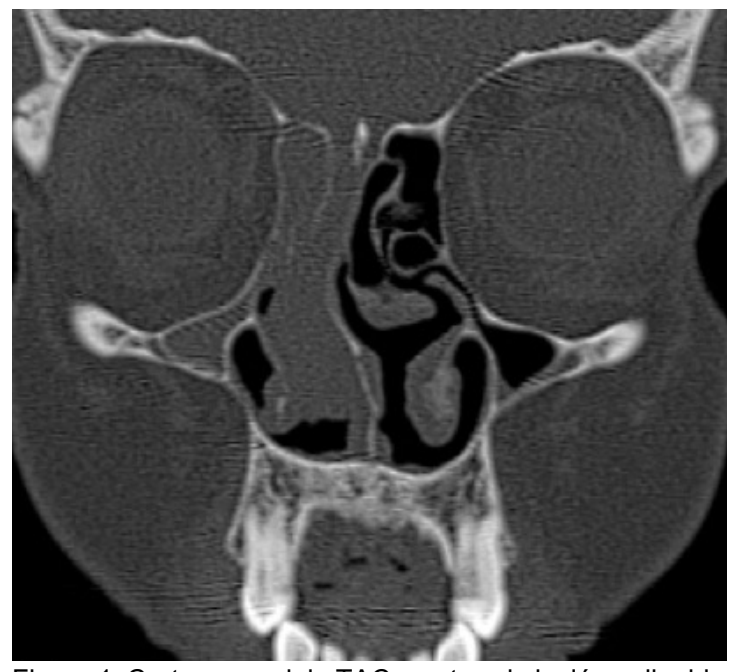

Figura 1. Corte coronal de TAC mostrando lesión polipoide unilateral.
Se realizó cirugía endoscópica nasal con fin diagnóstico y terapéutico consistente en polipectomía, meatotomía media y etmoidectomía anterior y posterior derechas, hallando poliposis y mucositis sin otros hallazgos.

El informe anatomopatológico reveló mucosa nasal infiltrada por proliferación celular en islotes de diferentes tamaños con patrón organoide con necrosis focal y separados por estroma fibroso vascularizado que presentaban células de tamaño intermedio con variabilidad en la forma y el tamaño con núcleos de cromatina granujienta y carioteca irregular con un recuento de 1-2 mitosis por 10 campos de gran aumento sin presencia de invasión linfovascular (Figura 2).

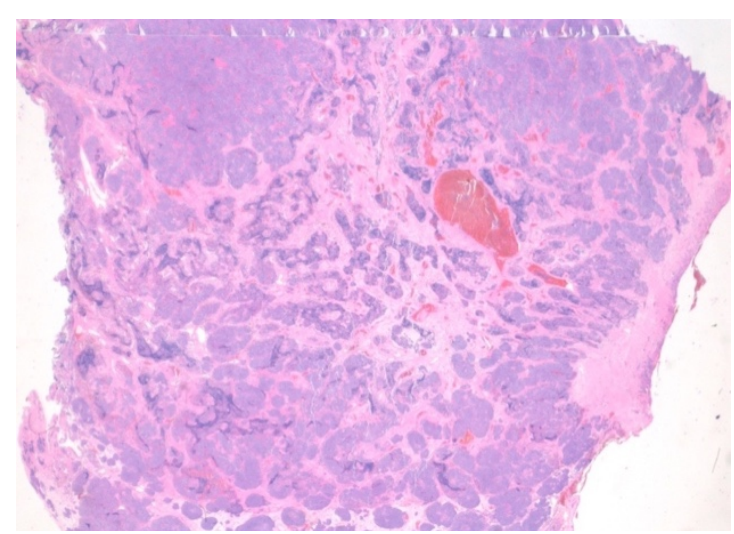

Figura 2. Histología de la tumoración que muestra patrón organoide.

El análisis inmunohistoquímico presentó intensa positividad con cromogranina y sinaptofisina y negatividad con CK5-6, p63, Vimentina y CKAE1-AE3. El índice de proliferación celular fue del 10\% con Ki67 (Figura 3).

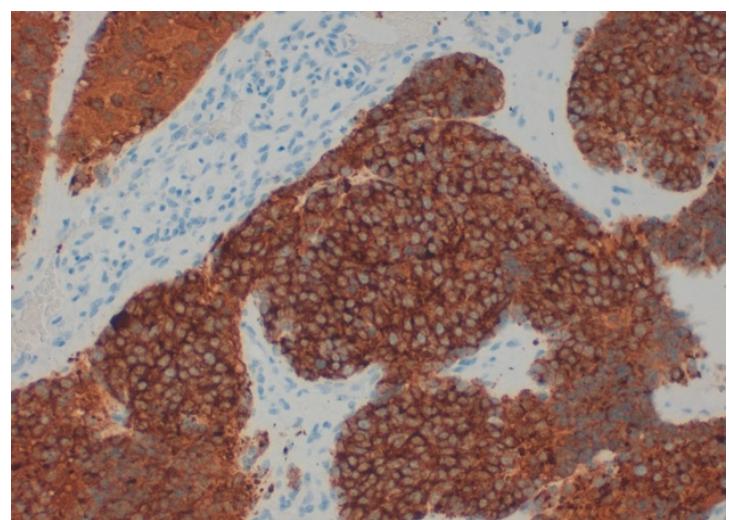

Figura 3. Análisis inmunohistoquímico: positividad con sinaptofisina.

(c) Ediciones Universidad de Salamanca / CC BY-NC-ND [104]

Rev. ORL, 2016, 7, 2, pp. 103-106 
Estos hallazgos permitieron hacer el diagnóstico de tumor carcinoide atípico o moderadamente diferenciado.

Se completó el estudio con analíticas para descartar síndrome carcinoide (serotonina sérica, noradrenalina, adrenalina, dopamina y ácido 5-hidroxi-indol-acético -5-HIAAT - en orina de 24 horas) que resultaron en límites normales. Se realizó TAC de cuerpo completo que descartó enfermedad en otras localizaciones y una resonancia magnética nuclear de senos paranasales con difusión-perfusión que evidenció restos de tumor en fosa nasal derecha y persistencia de cambios inflamatorios en seno frontal derecho.

La paciente fue intervenida nuevamente para revisión de cirugía endoscópica nasosinusal y realización de Lothrop modificado -DRAF III-, evidenciando mucositis en ambos senos frontales sin otros hallazgos valorables. La anatomía patológica en este caso no informó de tumoración residual.

\section{DISCUSIÓN}

Los tumores carcinoides se clasifican según los criterios de la Organización Mundial de la Salud (OMS) que inicialmente se propuso para tumores neuroendocrinos pulmonares. En 2005 en la nomenclatura de Otorrinolaringología (ORL) distingue cuatro tipos: tumor carcinoide, carcinoide atípico, tumor de células pequeñas y carcinoma combinado neuroendocrino de células pequeñas $[6,7]$. La OMS aún no considera para el área ORL el de células grandes como en los carcinomas pulmonares.

Se distinguen tumores bien diferenciados o típicos y moderadamente diferenciados o atípicos [1]. Los típicos presentan citología sin atipias, ausencia de necrosis y presentan menos de 4 mitosis por 10 campos de gran aumento. En cambio los atípicos presentan pocas atipias, necrosis aisladas y de 4 a 10 mitosis por 10 campos de gran aumento. Aunque es muy poco común, un tumor con pocas atipias pero abundante necrosis también se considera moderadamente diferenciado [2]. Los tumores típicos suelen ser de crecimiento lento y los atípicos o moderadamente diferenciados son más agresivos y pueden dar metástasis a distancia [3]. El análisis inmunohistoquímico de estos tumores revela positividad para marcadores de tejido neuroendocrino tales como cromogranina y sinaptofisina.
El análisis anatomopatológico del caso presentado describe necrosis, atipia ligera (núcleos no homogéneos y variables) y un índice de proliferación celular entre el $2-20 \%$ que permiten establecer el grado de diferenciación celular como moderadamente diferenciado (o atípico) a pesar de que el grado de mitosis es de 1 por 10 campos de gran aumento. La presentación en islotes y la cromatina granujienta (o en "rueda de carro») sugieren una diferenciación neuroendocrina que se confirma con la positividad con sinaptofisina y cromogranina. En la revisión bibliográfica realizada, solamente hemos encontrado 8 casos descritos en el seno esfenoidal y aproximadamente 120 en cavidad nasal y resto de senos paranasales; sin embargo es posible que su incidencia sea mayor ya que muchos de los casos podrían pasar desapercibidos, principalmente en los lugares de bajo acceso al sistema de salud y otros que pueden confundirse con adenocarcinoma, carcinoma indiferenciado u otras entidades neoplásicas.

El paciente con tumor carcinoide, independientemente de su localización, debe ser sometido a un estudio completo que incluya niveles de serotonina en sangre y de 5-HIAAT en orina de 24 horas para detectar tumores secretores y un estudio de imagen de cuerpo completo para detectar metástasis u otras localizaciones. Es importante destacar que no existen signos radiológicos específicos que permitan diferenciarlos de tumores de otra estirpe. Estudios recientes sugieren que la gammagrafía con receptores de somatostatina es la prueba de imagen de elección en el manejo de estas tumoraciones.

El tratamiento de los tumores carcinoides nasales, dada su baja frecuencia, no está bien establecido. Las opciones incluyen cirugía, radioterapia o quimioterapia con análogos de la somatostatina [4]. Se asume que el tratamiento del tumor primario localizado en cavidad nasal es esencialmente quirúrgico, como lo demuestran los escasos casos publicados, quedando la quimioterapia y la radioterapia confinada a los pacientes con metástasis, aquellos no buenos candidatos a la cirugía o cuando la resección quirúrgica se considera incompleta [5].

Debido a su baja incidencia, la supervivencia global de los pacientes con este tipo de tumores en cabeza y cuello es difícil de calcular. Soga [6] estima una supervivencia a cinco años del $46,7 \%$ en la localización laríngea [6] 
que es la más afectada y de la que más casos hay descritos en el área ORL [7-9].

\section{CONCLUSIONES}

El caso presentado destaca la importancia del estudio anatomopatológico de las lesiones polipoides unilaterales en cavidad nasal y senos paranasales. Consideramos que a pesar de ser una entidad infrecuente, con poca bibliografía y en la que no existen signos clínicos ni radiológicos específicos [9-11], el carcinoma neuroendocrino debe estar presente en el diagnóstico diferencial de los tumores primaros de senos paranasales.

\section{BIBLIOGRAFÍA}

1. Dhavala K, Vijayendra S, Sreenivas $\mathrm{N}$. Atypical carcinoid of larynx: A case study and a brief review. JCDR. 2014;8(4):KD03-KD04.

2. Bell D, Hanna E, Weber R, De Monte F, Triantafyllou $A$, Lewis $J$, et al. Neuroendocrine neoplasms of the sinonasal region. Head Neck. 2016 Apr;38 Suppl 1:E2259-66.

3. Stephenson K, Lubbe D. Primary atypical carcinoid tumour of the sphenoid sinus rostrum. Case Rep Otolaryngol [Internet]. 2014. Disponible en: http://www.hindawi.com/journals/criot/201 4/753964/. [Citado el 31 de marzo de 2016].

4. Chu M, Karakla D, Silverberg M, Han J. Primary carcinoid tumor of the frontal sinus: A case report. Ear Nose Throat J. 2010;89:13-6.

5. Mhawej R, Farah C, Haddad A, Tabchy B. Primary neuroendocrine tumors of the ear, nose and throat: A report of three cases and a review of the literature. Oncol Lett. 2015;10(4):2533-6.

6. Soga J. Carcinoids and their variant endocrinomas. An analysis of 11842 reported cases. J Exp Clin Cancer Res. 2003;22:517-30

7. Weinreb I, Pérez-Ordóñez B. Non-Small Cell neuroendocrine Carcinoma of the Sinonasal Tract and Nasopharynx. Report of 2 Cases and Review of the Literature. Head Neck Pathol. 2007;1:21-6.
8. Babin E, Rouleau V, Vedrine PO, Toussaint $B$, de Raucourt D, Malard O. Small cell neuroendocrine carcinoma of the nasal cavity and paranasal sinuses. J Laryngol Otol. 2006;120:289-97.

9. Smith SR, Som P, Fahmy A, Lawson W, Sacks S, Brandwein M. A Clinicopathological Study of Sinonasal Neuroendocrine Carcinoma and Sinonasal Undifferentiated Carcinoma. Laryngoscope. 2000;110:1617-22.

10. Uday SK, Prabhakar PK, Stan McH. Imaging of Nonlaryngeal Neuroendocrine Carcinoma. Am J Neuroradiol. 2000;21:775-8.

11. Marcos M, Landínez G, Martínez G, Morais D. Carcinomas neuroendocrinos en ORL: Un diagnóstico difícil. Acta Otorrinolaringol Esp. 2011;62(1):51-5. 